\section{Crystal structure of poly[ $(\mathrm{N}, \mathrm{N}$-dimethyl- acetamide- $\kappa O)\left(\mu_{4}-5\right.$-methylisophthalato- $\left.\kappa^{5} O: O, O^{\prime}: O^{\prime \prime}: O^{\prime \prime \prime}\right)$ manganese(II)]}

\author{
Lan Jin, Li-Li Zha, San Gao, Shi-Yao Yang* and Rong-Bin \\ Huang
}

Department of Chemistry, College of Chemistry and Chemical Engineering, Xiamen University, Xiamen 361005, People's Republic of China. *Correspondence e-mail: syyang@xmu.edu.cn

Received 19 November 2014; accepted 23 November 2014

Edited by U. Flörke, University of Paderborn, Germany

The title compound, poly $\left[(N, N\right.$-dimethylacetamide- $\kappa O)\left(\mu_{4^{-}}\right.$ 5-methylisophthalato- $\left.\kappa^{5} O, O^{\prime}: O^{\prime}, O^{\prime \prime}: O^{\prime \prime}\right)$ manganese(II)], $\left[\mathrm{Mn}\left(\mathrm{C}_{9} \mathrm{H}_{6} \mathrm{O}_{4}\right)\left(\mathrm{C}_{3} \mathrm{H}_{7} \mathrm{NO}\right)\right]_{n}$, was obtained from a mixture containing $\mathrm{MnCl}_{2} \cdot 4 \mathrm{H}_{2} \mathrm{O}$ and 5-methylisophthalic acid in $\mathrm{N}, \mathrm{N}$-dimethylacetamide solution. The $\mathrm{Mn}^{2+}$ ion is coordinated by five $\mathrm{O}$ atoms from four bridging 5-methylisophthalate ligands and one $\mathrm{O}$ atom from one $\mathrm{N}, \mathrm{N}$-dimethylacetamide ligand, defining a considerably distorted coordination polyhedron with one very long $\mathrm{Mn}-\mathrm{O}$ bond of 2.623 (2) $\AA$. The $\mathrm{Mn}^{2+}$ ions are joined by carboxylate groups, forming rodshaped secondary building units along the $a$ axis. The rods are further connected by 5 -methylisophthalate ligands to form the pcu (primitive cubic net) structure.

Keywords: crystal structure; manganese(II) coordination polymer; pcu structure; N,N-dimethylacetamide; 5-methylisophthalate.

CCDC reference: 1035658

\section{Related literature}

For the structures of coordination polymers comprising firstrow transition metal ions and benzene dicarboxylates, see: Deng et al. (2013); Jin et al. (2012); Li et al. (2010); Yang et al. (2013); Zhou et al. (2009). For the nomenclature for metalorganic frameworks, see: Rosi et al. (2005);. A very closely related crystal structure, poly[(dimethylformamide)(5-methoxybenzene-1,3-dicarboxylato)manganese(II)], was reported recently (Huang, 2013). The author described the structure in a PtS (cooperite) topology according to a different analytical approach (Carlucci et al., 2003; Hill et al., 2005).

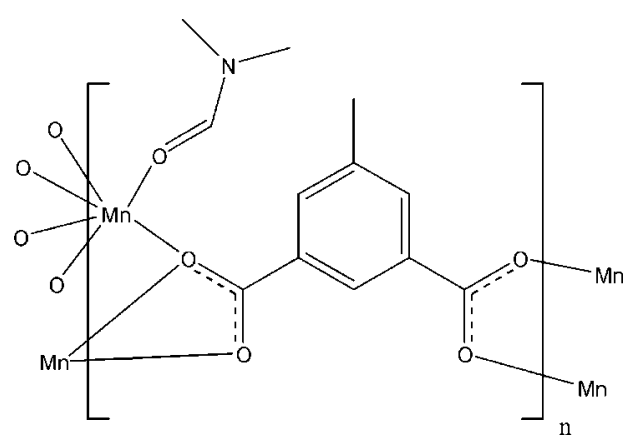

\section{Experimental}

\subsection{Crystal data}

$\left[\mathrm{Mn}\left(\mathrm{C}_{9} \mathrm{H}_{6} \mathrm{O}_{4}\right)\left(\mathrm{C}_{3} \mathrm{H}_{7} \mathrm{NO}\right)\right]$

$M_{r}=306.17$

Orthorhombic, Pna2 ${ }_{1}$

$a=7.281(5) \AA$

$b=15.148(11) \AA$

$c=10.903(8) \AA$

$$
\begin{aligned}
& V=1202.5(15) \AA^{3} \\
& Z=4 \\
& \text { Mo } K \alpha \text { radiation } \\
& \mu=1.11 \mathrm{~mm}^{-1} \\
& T=200 \mathrm{~K} \\
& 0.15 \times 0.10 \times 0.10 \mathrm{~mm}
\end{aligned}
$$

\subsection{Data collection}

Bruker APEX area-detector diffractometer

Absorption correction: multi-scan (SADABS; Sheldrick, 2004)

$T_{\min }=0.851, T_{\max }=0.897$

10159 measured reflections 2874 independent reflections 2768 reflections with $I>2 \sigma(I)$ $R_{\text {int }}=0.025$

\subsection{Refinement}

$\begin{array}{lc}R\left[F^{2}>2 \sigma\left(F^{2}\right)\right]=0.024 & \Delta \rho_{\max }=0.26 \mathrm{e} \AA^{-3} \\ w R\left(F^{2}\right)=0.059 & \Delta \rho_{\min }=-0.29 \AA^{-3} \\ S=1.07 & \text { Absolute structure: Flack (1983), } \\ 2874 \text { reflections } & \text { 0 Friedel pairs } \\ \text { 175 parameters } & \text { Absolute structure parameter: } \\ 1 \text { restraint } & 0.025(14) \\ \text { H-atom parameters constrained } & \end{array}$

Table 1

Selected geometric parameters $\left(\AA{ }^{\circ}\right)$.

\begin{tabular}{lrlr}
\hline $\mathrm{Mn} 1-\mathrm{O}^{\mathrm{i}}$ & $2.0609(19)$ & $\mathrm{Mn} 1-\mathrm{O} 5$ & $2.1342(18)$ \\
$\mathrm{Mn} 1-3^{\mathrm{ii}}$ & $2.0855(15)$ & $\mathrm{Mn} 1-\mathrm{O} 2^{\mathrm{iii}}$ & $2.1378(16)$ \\
$\mathrm{Mn} 1-\mathrm{O} 1$ & $2.0885(16)$ & & \\
$\mathrm{O}^{\mathrm{i}}-\mathrm{Mn} 1-\mathrm{O}^{\mathrm{ii}}$ & $131.59(6)$ & $\mathrm{O} 1-\mathrm{Mn} 1-\mathrm{O} 5$ & $166.04(5)$ \\
$\mathrm{O}^{\mathrm{i}}-\mathrm{Mn} 1-\mathrm{O} 1$ & $83.59(6)$ & $\mathrm{O} 4^{\mathrm{i}}-\mathrm{Mn} 1-\mathrm{O} 2^{\mathrm{iii}}$ & $135.70(6)$ \\
$\mathrm{O}^{\mathrm{ii}}-\mathrm{Mn} 1-\mathrm{O} 1$ & $98.77(7)$ & $\mathrm{O} 3^{\mathrm{ii}}-\mathrm{Mn} 1-\mathrm{O} 2^{\mathrm{iii}}$ & $92.23(7)$ \\
$\mathrm{O}^{\mathrm{i}}-\mathrm{Mn} 1-\mathrm{O} 5$ & $83.95(6)$ & $\mathrm{O} 1-\mathrm{Mn} 1-\mathrm{O} 2^{\mathrm{iii}}$ & $97.51(7)$ \\
$\mathrm{O}^{\mathrm{ii}}-\mathrm{Mn} 1-\mathrm{O} 5$ & $84.88(7)$ & $\mathrm{O} 5-\mathrm{Mn} 1-\mathrm{O} 2^{\mathrm{iii}}$ & $95.80(7)$ \\
\hline
\end{tabular}

Symmetry codes: (i) $-x,-y+2, z+\frac{1}{2}$; (ii) $-x+\frac{1}{2}, y-\frac{1}{2}, z+\frac{1}{2}$; (iii) $x-\frac{1}{2},-y+\frac{3}{2}, z$.

Data collection: SMART (Bruker, 2002); cell refinement: SAINT (Bruker, 2002); data reduction: $S A I N T$; $\operatorname{program}(\mathrm{s})$ used to solve structure: SHELXTL (Sheldrick, 2008); program(s) used to refine structure: SHELXTL; molecular graphics: DIAMOND (Brandenburg, 2007); software used to prepare material for publication: SHELXTL. 


\section{Acknowledgements}

We are grateful for financial support by the National Natural Science Foundation of China (grant Nos. 21071117 and 21471125).

Supporting information for this paper is available from the IUCr electronic archives (Reference: FK2084).

\section{References}

Brandenburg, K. (2007). DIAMOND. Crystal Impact GbR, Bonn, Germany. Bruker (2002). SMART and SAINT. Bruker AXS Inc., Madison, Wisconsin, USA.

Carlucci, L., Ciani, G. \& Proserpio, D. M. (2003). Coord. Chem. Rev. 246, 247289.
Deng, X.-L., Yang, S.-Y., Jin, R.-F., Tao, J., Wu, C.-Q., Li, Z.-L., Long, L.-S., Huang, R.-B. \& Zheng, L.-S. (2013). Polyhedron, 50, 219-228.

Flack, H. D. (1983). Acta Cryst. A39, 876-881.

Hill, R. J., Long, D.-L., Champness, N. R., Hubberstey, P. \& Schröder, M. (2005). Acc. Chem. Res. 38, 337-350.

Huang, X.-H. (2013). Acta Cryst. C69, 483-485.

Jin, R.-F., Yang, S.-Y., Li, H.-M., Long, L.-S., Huang, R.-B. \& Zheng, L.-S. (2012). CrystEngComm, 14, 1301-1316.

Li, H.-M., Yang, S.-Y., Wang, J.-W., Long, L.-S., Huang, R.-B. \& Zheng, L.-S. (2010). Polyhedron, 29, 2851-2856.

Rosi, N. L., Kim, J., Eddaoudi, M., Chen, B., O’Keeffe, M. \& Yaghi, O. M. (2005). J. Am. Chem. Soc. 127, 1504-1518.

Sheldrick, G. M. (2004). SADABS. University of Göttingen, Germany.

Sheldrick, G. M. (2008). Acta Cryst. A64, 112-122.

Yang, S.-Y., Yuan, H.-B., Xu, X.-B. \& Huang, R.-B. (2013). Inorg. Chim. Acta, 403, 53-62.

Zhou, D.-S., Wang, F.-K., Yang, S.-Y., Xie, Z.-X. \& Huang, R.-B. (2009). CrystEngComm, 11, 2548-2554. 


\section{supporting information}

Acta Cryst. (2015). E71, m1-m2［https://doi.org/10.1107/S2056989014025626]

\section{Crystal structure of poly $\left[(\mathrm{N}, \mathrm{N}\right.$-dimethylacetamide- $\kappa \mathrm{O})\left(\mu_{4}-5\right.$-methylisophthalato- $\left.\kappa^{5} O: O, O^{\prime}: O^{\prime \prime}: O^{\prime \prime \prime}\right)$ manganese(II)]}

\section{Lan Jin, Li-Li Zha, San Gao, Shi-Yao Yang and Rong-Bin Huang}

\section{S1. Structural commentary}

We have reported the structures of dozens of coordination polymers comprising first-row transition metal ions and benzene dicarboxylates (Deng et al., 2013; Jin et al., 2012; Li et al. 2010; Yang et al., 2013; Zhou et al., 2009). We found that metal ion is the most important factor that influences the structure of coordination polymer.

The title compound, [ $\left.\mathrm{Mn}\left(\mathrm{C}_{9} \mathrm{H}_{6} \mathrm{O}_{4}\right)\left(\mathrm{C}_{3} \mathrm{H}_{7} \mathrm{O}\right)\right]_{\mathrm{n}}$, (I) was obtained with the same method reported in our previous paper

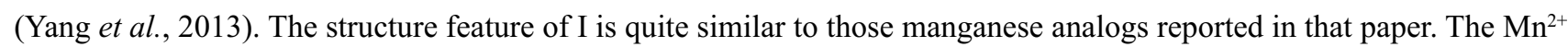
ions are joined by carboxyl groups to form rod-shaped secondary building units (SBUs) along the $a$ axis. Each rod is further connected to four adjacent rods by 5 -methylisophthalates to form the rod packing type 2 pcu (primitive cubic net) structure according to the nomenclature for metal-organic frameworks (Rosi et al., 2005). A very closely related molecular structure, poly[(dimethylformamide)(5-methoxybenzene-1,3-dicarboxylato)manganese(II)], was reported recently (Huang, 2013). The author described the structure in PtS (cooperite) topology according to a different analysis approach (Carlucci et al., 2003; Hill et al., 2005).

\section{S2. Synthesis and crystallization}

A mixture containing $\mathrm{MnCl}_{2} .4 \mathrm{H}_{2} \mathrm{O}(0.039 \mathrm{~g}, 0.20 \mathrm{mmol})$ and 5-methylisophthalic acid $\left(\mathrm{H}_{2} \mathrm{mip}, 0.036 \mathrm{~g}, 0.20 \mathrm{mmol}\right)$ in 10

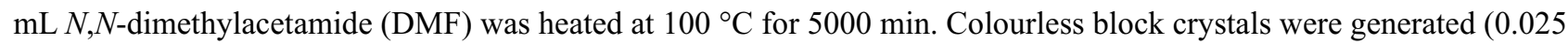
g, 41\%).

\section{S3. Refinement}

Crystal data, data collection and structure refinement details are summarized in Table $1 . \mathrm{H}$ atoms bonded to $\mathrm{C}$ atoms were positioned geometrically and refined using a riding model (including free rotation about the $\mathrm{C}-\mathrm{C}$ bond), with $\mathrm{C}-\mathrm{H}=$ $0.95-0.99 \AA$ and with $U_{\text {iso }}(\mathrm{H})=1.2\left(1.5\right.$ for methyl groups) times $U_{\text {eq }}(\mathrm{C})$. 


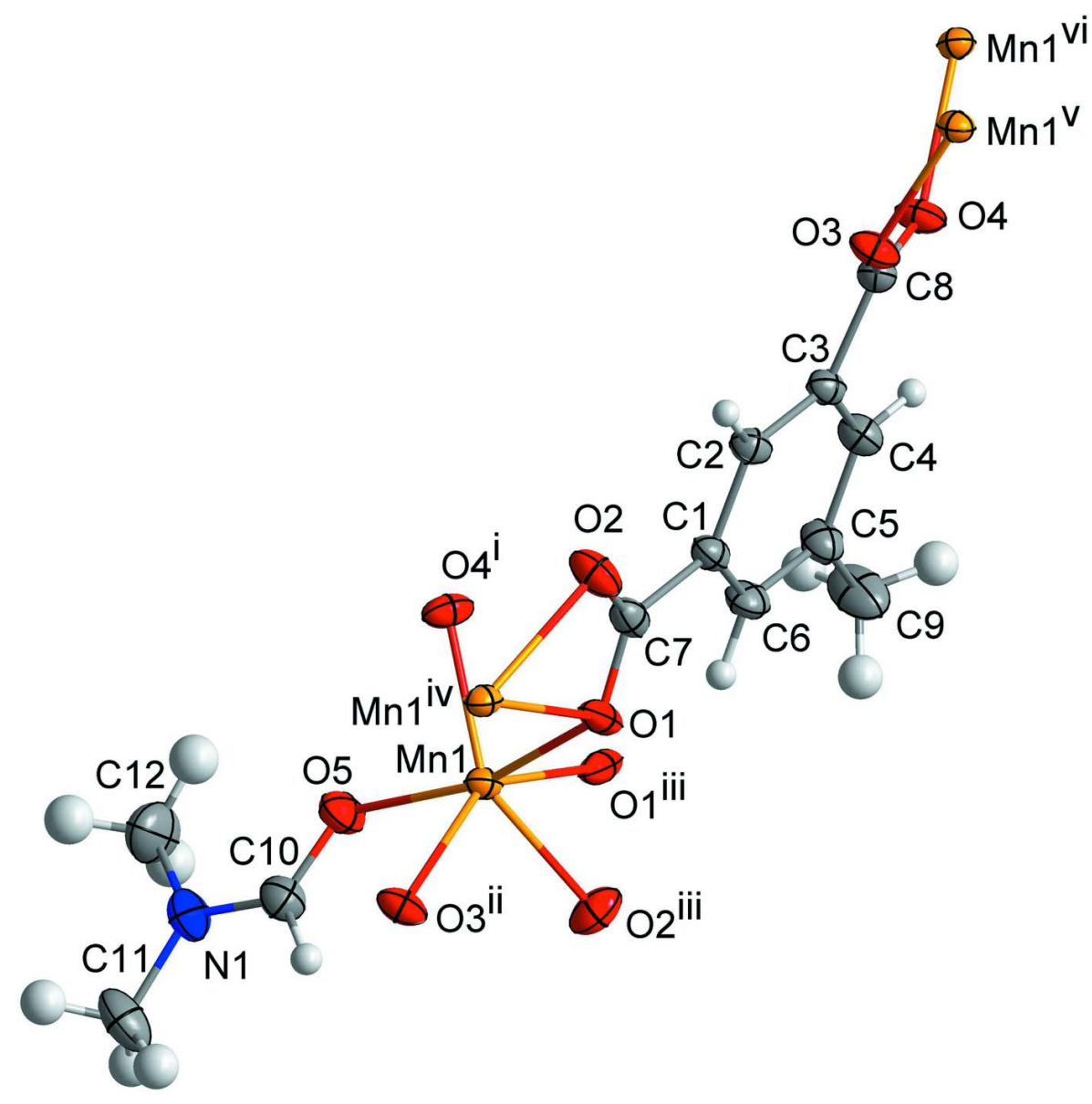

\section{Figure 1}

Coordination modes in (I). Anisotropic displacement ellipsoids are drawn at the $50 \%$ probability level. Symmetry codes: $\mathrm{i}$ $-x,-y+2, z+1 / 2$; ii $-x+1 / 2, y-1 / 2, z+1 / 2$; iii $x-1 / 2,-y+3 / 2, z$; iv $x+1 / 2,-y+3 / 2, z$; v $-x+1 / 2, y+1 / 2, z-1 / 2$; vi $-x$, $-y+2, z-1 / 2$. 


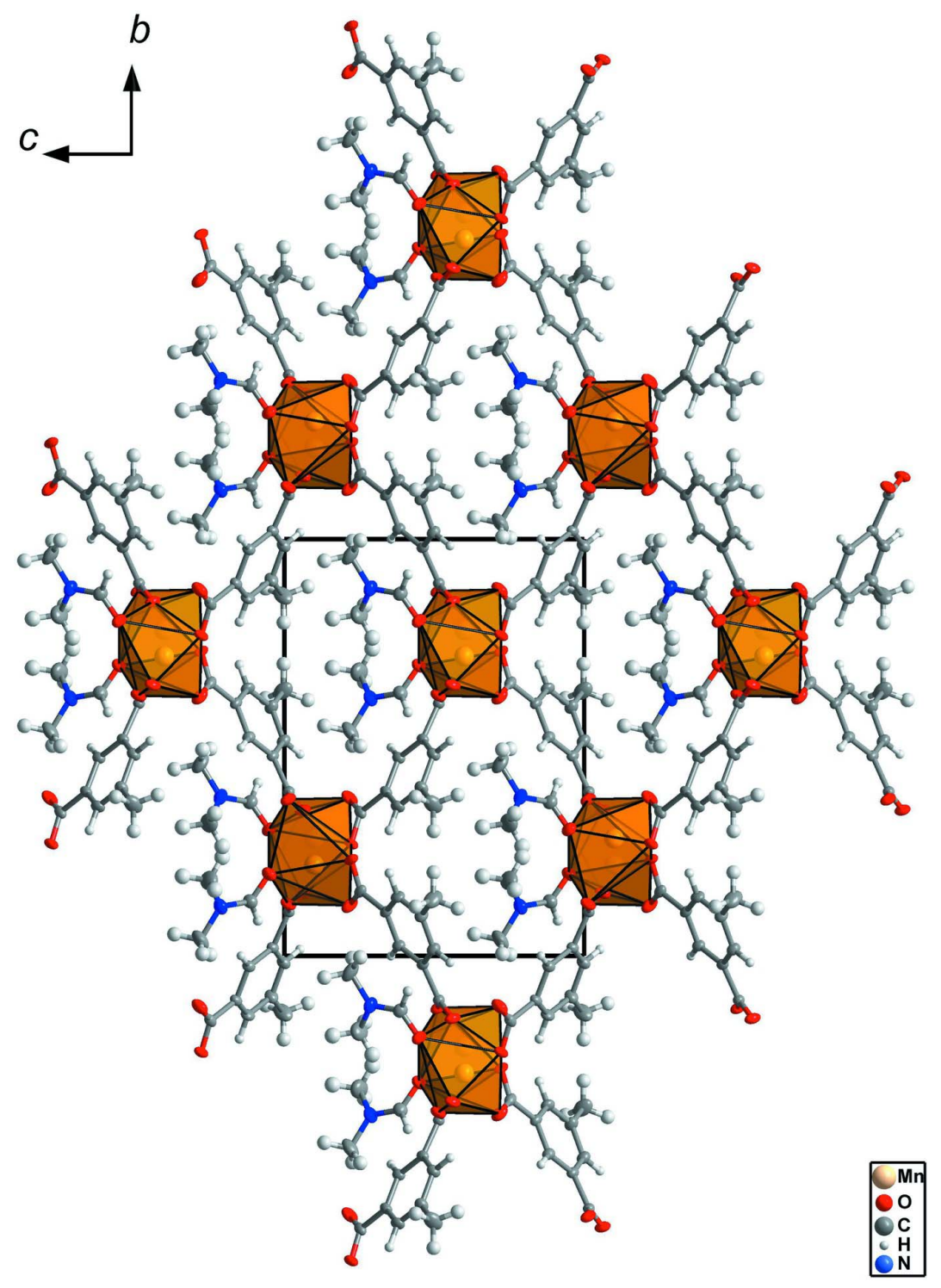

Figure 2

The packing of (I), viewed down the $a$ axis, showing $\mathrm{MnO}_{6}$ in polyhedra.

Poly $\left[(N, N\right.$-dimethylacetamide- $\kappa O)\left(\mu_{4}-5\right.$-methylisophthalato- $\left.\kappa^{5} O: O, O^{\prime}: O^{\prime \prime}: O^{\prime \prime \prime}\right)$ manganese(II)]

Crystal data

$\left[\mathrm{Mn}\left(\mathrm{C}_{9} \mathrm{H}_{6} \mathrm{O}_{4}\right)\left(\mathrm{C}_{3} \mathrm{H}_{7} \mathrm{NO}\right)\right]$

$M_{r}=306.17$

Orthorhombic, Pna2 $_{1}$

Hall symbol: $\mathrm{P} 2 \mathrm{c}-2 \mathrm{n}$

$a=7.281(5) \AA$

$b=15.148(11) \AA$

$c=10.903(8) \AA$

$V=1202.5(15) \AA^{3}$

$Z=4$

$F(000)=628$

$D_{\mathrm{x}}=1.691 \mathrm{Mg} \mathrm{m}^{-3}$

Mo $K \alpha$ radiation, $\lambda=0.71073 \AA$

Cell parameters from 6647 reflections

$\theta=2.3-28.7^{\circ}$

$\mu=1.11 \mathrm{~mm}^{-1}$

$T=200 \mathrm{~K}$

Rod, colorless

$0.15 \times 0.10 \times 0.10 \mathrm{~mm}$ 


\section{Data collection}

Bruker APEX area-detector diffractometer

Radiation source: fine-focus sealed tube Graphite monochromator $\varphi$ and $\omega$ scan

Absorption correction: multi-scan (SADABS; Sheldrick, 2004)

$T_{\min }=0.851, T_{\max }=0.897$

\section{Refinement}

Refinement on $F^{2}$

Least-squares matrix: full

$R\left[F^{2}>2 \sigma\left(F^{2}\right)\right]=0.024$

$w R\left(F^{2}\right)=0.059$

$S=1.07$

2874 reflections

175 parameters

1 restraint

Primary atom site location: structure-invariant direct methods
10159 measured reflections

2874 independent reflections

2768 reflections with $I>2 \sigma(I)$

$R_{\text {int }}=0.025$

$\theta_{\text {max }}=29.0^{\circ}, \theta_{\text {min }}=2.3^{\circ}$

$h=-9 \rightarrow 9$

$k=-19 \rightarrow 19$

$l=-13 \rightarrow 14$

Secondary atom site location: difference Fourier map

Hydrogen site location: inferred from neighbouring sites

$\mathrm{H}$-atom parameters constrained

$w=1 /\left[\sigma^{2}\left(F_{\mathrm{o}}^{2}\right)+(0.0316 P)^{2}+0.1079 P\right]$

where $P=\left(F_{\mathrm{o}}^{2}+2 F_{\mathrm{c}}^{2}\right) / 3$

$(\Delta / \sigma)_{\max }=0.001$

$\Delta \rho_{\max }=0.26$ e $\AA^{-3}$

$\Delta \rho_{\text {min }}=-0.29$ e $\AA^{-3}$

Absolute structure: Flack (1983), 0 Friedel pairs

Absolute structure parameter: 0.025 (14)

\section{Special details}

Geometry. All e.s.d.'s (except the e.s.d. in the dihedral angle between two 1.s. planes) are estimated using the full covariance matrix. The cell e.s.d.'s are taken into account individually in the estimation of e.s.d.'s in distances, angles and torsion angles; correlations between e.s.d.'s in cell parameters are only used when they are defined by crystal symmetry. An approximate (isotropic) treatment of cell e.s.d.'s is used for estimating e.s.d.'s involving 1.s. planes.

Refinement. Refinement of $F^{2}$ against ALL reflections. The weighted $R$-factor $w R$ and goodness of fit $S$ are based on $F^{2}$, conventional $R$-factors $R$ are based on $F$, with $F$ set to zero for negative $F^{2}$. The threshold expression of $F^{2}>\sigma\left(F^{2}\right)$ is used only for calculating $R$-factors(gt) etc. and is not relevant to the choice of reflections for refinement. $R$-factors based on $F^{2}$ are statistically about twice as large as those based on $F$, and $R$ - factors based on ALL data will be even larger.

Fractional atomic coordinates and isotropic or equivalent isotropic displacement parameters $\left(\AA^{2}\right)$

\begin{tabular}{lllll}
\hline & $x$ & $y$ & $z$ & $U_{\text {iso }} * / U_{\text {eq }}$ \\
\hline Mn1 & $0.02668(3)$ & $0.718473(13)$ & $0.40075(4)$ & $0.01733(7)$ \\
O1 & $0.21890(19)$ & $0.76885(7)$ & $0.27699(13)$ & $0.0264(3)$ \\
O2 & $0.40794(19)$ & $0.87833(8)$ & $0.28213(14)$ & $0.0320(3)$ \\
O3 & $0.30783(16)$ & $1.12474(7)$ & $-0.01315(13)$ & $0.0244(3)$ \\
O4 & $0.02059(16)$ & $1.15017(8)$ & $-0.06146(13)$ & $0.0254(3)$ \\
O5 & $-0.15683(19)$ & $0.69631(9)$ & $0.55025(14)$ & $0.0301(3)$ \\
N1 & $-0.2451(2)$ & $0.61847(11)$ & $0.71232(16)$ & $0.0323(4)$ \\
C1 & $0.1457(2)$ & $0.89377(11)$ & $0.16063(16)$ & $0.0210(3)$ \\
C2 & $0.1976(2)$ & $0.97512(10)$ & $0.11805(18)$ & $0.0208(3)$ \\
H2A & 0.3104 & 1.0003 & 0.1442 & $0.025^{*}$ \\
C3 & $0.0878(2)$ & $1.02002(10)$ & $0.03817(16)$ & $0.0196(3)$ \\
C4 & $-0.0754(2)$ & $0.98383(11)$ & $0.00205(17)$ & $0.0237(3)$ \\
H4A & -0.1529 & 1.0157 & -0.0523 & $0.028^{*}$ \\
C5 & $-0.1290(3)$ & $0.90257(12)$ & $0.04273(18)$ & $0.0269(4)$ \\
C6 & $-0.0157(3)$ & $0.85839(12)$ & $0.12133(19)$ & $0.0261(4)$
\end{tabular}


supporting information

\begin{tabular}{lllll} 
H6A & -0.0505 & 0.8014 & 0.1494 & $0.031^{*}$ \\
C7 & $0.2643(2)$ & $0.84485(11)$ & $0.24625(17)$ & $0.0236(4)$ \\
C8 & $0.1438(2)$ & $1.10570(10)$ & $-0.01549(17)$ & $0.0197(3)$ \\
C9 & $-0.3076(3)$ & $0.86426(15)$ & $0.0039(3)$ & $0.0448(6)$ \\
H9A & -0.2980 & 0.7997 & 0.0020 & $0.067^{*}$ \\
H9B & -0.3392 & 0.8861 & -0.0780 & $0.067^{*}$ \\
H9C & -0.4034 & 0.8817 & 0.0623 & $0.067^{*}$ \\
C10 & $-0.1507(3)$ & $0.63087(12)$ & $0.61313(19)$ & $0.0282(4)$ \\
H10A & -0.0704 & 0.5849 & 0.5880 & $0.034^{*}$ \\
C11 & $-0.2297(3)$ & $0.53842(16)$ & $0.7799(2)$ & $0.0459(6)$ \\
H11A & -0.1283 & 0.5032 & 0.7469 & $0.069^{*}$ \\
H11B & -0.3446 & 0.5050 & 0.7731 & $0.069^{*}$ \\
H11C & -0.2057 & 0.5520 & 0.8664 & $0.069^{*}$ \\
C12 & $-0.3792(4)$ & $0.68114(17)$ & $0.7505(3)$ & $0.0545(7)$ \\
H12A & -0.3596 & 0.7369 & 0.7069 & $0.082^{*}$ \\
H12B & -0.3680 & 0.6911 & 0.8390 & $0.082^{*}$ \\
H12C & -0.5022 & 0.6586 & 0.7319 & $0.082^{*}$ \\
\hline
\end{tabular}

Atomic displacement parameters $\left(\AA^{2}\right)$

\begin{tabular}{lllllll}
\hline & $U^{11}$ & $U^{22}$ & $U^{33}$ & $U^{12}$ & $U^{13}$ & $U^{23}$ \\
\hline $\mathrm{Mn} 1$ & $0.01845(11)$ & $0.01338(10)$ & $0.02015(12)$ & $0.00142(7)$ & $0.00145(16)$ & $0.00084(12)$ \\
O1 & $0.0343(7)$ & $0.0170(5)$ & $0.0279(7)$ & $0.0074(5)$ & $0.0119(6)$ & $0.0068(5)$ \\
O2 & $0.0262(7)$ & $0.0330(7)$ & $0.0368(8)$ & $-0.0002(6)$ & $-0.0060(6)$ & $0.0145(6)$ \\
O3 & $0.0188(6)$ & $0.0207(5)$ & $0.0336(7)$ & $-0.0019(4)$ & $0.0012(5)$ & $0.0087(5)$ \\
O4 & $0.0232(6)$ & $0.0172(6)$ & $0.0359(8)$ & $0.0006(4)$ & $-0.0074(5)$ & $0.0059(5)$ \\
O5 & $0.0333(7)$ & $0.0266(6)$ & $0.0305(8)$ & $0.0002(6)$ & $0.0079(6)$ & $0.0069(6)$ \\
N1 & $0.0328(9)$ & $0.0366(9)$ & $0.0274(10)$ & $-0.0036(7)$ & $0.0051(7)$ & $0.0065(7)$ \\
C1 & $0.0227(8)$ & $0.0191(7)$ & $0.0211(8)$ & $0.0021(6)$ & $0.0043(6)$ & $0.0043(6)$ \\
C2 & $0.0192(8)$ & $0.0193(7)$ & $0.0240(8)$ & $0.0002(6)$ & $0.0026(6)$ & $0.0021(6)$ \\
C3 & $0.0202(8)$ & $0.0175(7)$ & $0.0212(8)$ & $0.0010(6)$ & $0.0047(7)$ & $0.0019(6)$ \\
C4 & $0.0217(8)$ & $0.0230(8)$ & $0.0264(9)$ & $0.0006(6)$ & $0.0005(7)$ & $0.0053(7)$ \\
C5 & $0.0267(9)$ & $0.0274(9)$ & $0.0267(9)$ & $-0.0067(7)$ & $-0.0001(7)$ & $0.0044(7)$ \\
C6 & $0.0295(9)$ & $0.0201(8)$ & $0.0286(10)$ & $-0.0051(6)$ & $0.0048(7)$ & $0.0040(7)$ \\
C7 & $0.0262(8)$ & $0.0235(8)$ & $0.0213(9)$ & $0.0070(7)$ & $0.0060(7)$ & $0.0051(7)$ \\
C8 & $0.0223(8)$ & $0.0168(7)$ & $0.0199(8)$ & $0.0008(6)$ & $0.0000(6)$ & $0.0005(6)$ \\
C9 & $0.0371(12)$ & $0.0452(12)$ & $0.0521(14)$ & $-0.0188(9)$ & $-0.0127(10)$ & $0.0132(11)$ \\
C10 & $0.0253(9)$ & $0.0291(9)$ & $0.0303(10)$ & $-0.0008(7)$ & $0.0022(8)$ & $0.0026(8)$ \\
C11 & $0.0445(13)$ & $0.0526(13)$ & $0.0407(14)$ & $-0.0074(10)$ & $0.0018(11)$ & $0.0222(11)$ \\
C12 & $0.0674(17)$ & $0.0524(14)$ & $0.0437(15)$ & $0.0092(13)$ & $0.0238(13)$ & $-0.0037(12)$ \\
& & & & & & \\
\hline
\end{tabular}

Geometric parameters $\left(\AA,{ }^{\circ}\right)$

\begin{tabular}{llll}
\hline $\mathrm{Mn} 1-\mathrm{O} 4^{\mathrm{i}}$ & $2.0609(19)$ & $\mathrm{C} 2-\mathrm{C} 3$ & $1.364(2)$ \\
$\mathrm{Mn} 1-\mathrm{O} 3^{\text {ii }}$ & $2.0855(15)$ & $\mathrm{C} 2-\mathrm{H} 2 \mathrm{~A}$ & 0.9500 \\
$\mathrm{Mn} 1-\mathrm{O} 1$ & $2.0885(16)$ & $\mathrm{C} 3-\mathrm{C} 4$ & $1.367(2)$ \\
$\mathrm{Mn} 1-\mathrm{O} 5$ & $2.1342(18)$ & $\mathrm{C} 3-\mathrm{C} 8$ & $1.481(2)$ \\
$\mathrm{Mn} 1-\mathrm{O} 2^{\mathrm{iii}}$ & $2.1378(16)$ & $\mathrm{C} 4-\mathrm{C} 5$ & $1.365(2)$
\end{tabular}




\begin{tabular}{|c|c|c|c|}
\hline $\mathrm{O} 1-\mathrm{C} 7$ & $1.244(2)$ & $\mathrm{C} 4-\mathrm{H} 4 \mathrm{~A}$ & 0.9500 \\
\hline $\mathrm{O} 2-\mathrm{C} 7$ & $1.226(2)$ & $\mathrm{C} 5-\mathrm{C} 6$ & $1.365(3)$ \\
\hline $\mathrm{O} 2-\mathrm{Mn} 1^{\mathrm{iv}}$ & $2.1378(16)$ & $\mathrm{C} 5-\mathrm{C} 9$ & $1.485(3)$ \\
\hline $\mathrm{O} 3-\mathrm{C} 8$ & $1.229(2)$ & C6-H6A & 0.9500 \\
\hline $\mathrm{O} 3-\mathrm{Mn} 1^{\mathrm{v}}$ & $2.0855(15)$ & C9-H9A & 0.9800 \\
\hline $\mathrm{O} 4-\mathrm{C} 8$ & $1.228(2)$ & С9-H9B & 0.9800 \\
\hline $\mathrm{O} 4-\mathrm{Mn} 1^{\mathrm{vi}}$ & 2.0609 (18) & $\mathrm{C} 9-\mathrm{H} 9 \mathrm{C}$ & 0.9800 \\
\hline $\mathrm{O} 5-\mathrm{C} 10$ & $1.206(2)$ & $\mathrm{C} 10-\mathrm{H} 10 \mathrm{~A}$ & 0.9500 \\
\hline $\mathrm{N} 1-\mathrm{C} 10$ & $1.295(3)$ & C11-H11A & 0.9800 \\
\hline $\mathrm{N} 1-\mathrm{C} 11$ & $1.424(3)$ & $\mathrm{C} 11-\mathrm{H} 11 \mathrm{~B}$ & 0.9800 \\
\hline $\mathrm{N} 1-\mathrm{C} 12$ & $1.424(3)$ & $\mathrm{C} 11-\mathrm{H} 11 \mathrm{C}$ & 0.9800 \\
\hline $\mathrm{C} 1-\mathrm{C} 6$ & $1.361(3)$ & $\mathrm{C} 12-\mathrm{H} 12 \mathrm{~A}$ & 0.9800 \\
\hline $\mathrm{C} 1-\mathrm{C} 2$ & $1.370(2)$ & $\mathrm{C} 12-\mathrm{H} 12 \mathrm{~B}$ & 0.9800 \\
\hline $\mathrm{C} 1-\mathrm{C} 7$ & $1.472(2)$ & $\mathrm{C} 12-\mathrm{H} 12 \mathrm{C}$ & 0.9800 \\
\hline $\mathrm{O} 4^{\mathrm{i}}-\mathrm{Mn} 1-\mathrm{O} 3^{\mathrm{ii}}$ & $131.59(6)$ & $\mathrm{C} 4-\mathrm{C} 5-\mathrm{C} 9$ & $120.65(19)$ \\
\hline $\mathrm{O} 4^{\mathrm{i}}-\mathrm{Mn} 1-\mathrm{O} 1$ & $83.59(6)$ & $\mathrm{C} 1-\mathrm{C} 6-\mathrm{C} 5$ & $121.77(17)$ \\
\hline $\mathrm{O} 33^{\mathrm{ii}}-\mathrm{Mn} 1-\mathrm{O} 1$ & $98.77(7)$ & $\mathrm{C} 1-\mathrm{C} 6-\mathrm{H} 6 \mathrm{~A}$ & 119.1 \\
\hline $\mathrm{O} 44^{\mathrm{i}}-\mathrm{Mn} 1-\mathrm{O} 5$ & $83.95(6)$ & $\mathrm{C} 5-\mathrm{C} 6-\mathrm{H} 6 \mathrm{~A}$ & 119.1 \\
\hline $\mathrm{O} 3^{\mathrm{ii}}-\mathrm{Mn} 1-\mathrm{O} 5$ & $84.88(7)$ & $\mathrm{O} 2-\mathrm{C} 7-\mathrm{O} 1$ & $121.56(17)$ \\
\hline $\mathrm{O} 1-\mathrm{Mn} 1-\mathrm{O} 5$ & $166.04(5)$ & $\mathrm{O} 2-\mathrm{C} 7-\mathrm{C} 1$ & $119.62(16)$ \\
\hline $\mathrm{O} 4-\mathrm{Mn} 1-\mathrm{O} 2^{\mathrm{iii}}$ & $135.70(6)$ & $\mathrm{O} 1-\mathrm{C} 7-\mathrm{C} 1$ & $118.76(17)$ \\
\hline $\mathrm{O} 3^{\mathrm{ii}}-\mathrm{Mn} 1-\mathrm{O} 2^{\mathrm{iii}}$ & $92.23(7)$ & $\mathrm{O} 4-\mathrm{C} 8-\mathrm{O} 3$ & $126.10(17)$ \\
\hline $\mathrm{O} 1-\mathrm{Mn} 1-\mathrm{O} 2^{\mathrm{iii}}$ & $97.51(7)$ & $\mathrm{O} 4-\mathrm{C} 8-\mathrm{C} 3$ & $116.16(16)$ \\
\hline $\mathrm{O} 5-\mathrm{Mn} 1-\mathrm{O} 2^{\mathrm{iii}}$ & $95.80(7)$ & $\mathrm{O} 3-\mathrm{C} 8-\mathrm{C} 3$ & $117.70(15)$ \\
\hline $\mathrm{C} 7-\mathrm{O} 1-\mathrm{Mn} 1$ & $133.66(12)$ & $\mathrm{C} 5-\mathrm{C} 9-\mathrm{H} 9 \mathrm{~A}$ & 109.5 \\
\hline $\mathrm{C} 7-\mathrm{O} 2-\mathrm{Mn} 1^{\mathrm{iv}}$ & $104.72(11)$ & $\mathrm{C} 5-\mathrm{C} 9-\mathrm{H} 9 \mathrm{~B}$ & 109.5 \\
\hline $\mathrm{C} 8-\mathrm{O} 3-\mathrm{Mn} 1^{\mathrm{v}}$ & $135.39(11)$ & $\mathrm{H} 9 \mathrm{~A}-\mathrm{C} 9-\mathrm{H} 9 \mathrm{~B}$ & 109.5 \\
\hline $\mathrm{C} 8-\mathrm{O} 4-\mathrm{Mn} 1^{\mathrm{vi}}$ & $137.13(12)$ & $\mathrm{C} 5-\mathrm{C} 9-\mathrm{H} 9 \mathrm{C}$ & 109.5 \\
\hline $\mathrm{C} 10-\mathrm{O} 5-\mathrm{Mn} 1$ & $122.70(13)$ & $\mathrm{H} 9 \mathrm{~A}-\mathrm{C} 9-\mathrm{H} 9 \mathrm{C}$ & 109.5 \\
\hline $\mathrm{C} 10-\mathrm{N} 1-\mathrm{C} 11$ & 120.94 (19) & $\mathrm{H} 9 \mathrm{~B}-\mathrm{C} 9-\mathrm{H} 9 \mathrm{C}$ & 109.5 \\
\hline $\mathrm{C} 10-\mathrm{N} 1-\mathrm{C} 12$ & $120.75(18)$ & $\mathrm{O} 5-\mathrm{C} 10-\mathrm{N} 1$ & $125.04(18)$ \\
\hline $\mathrm{C} 11-\mathrm{N} 1-\mathrm{C} 12$ & $118.1(2)$ & $\mathrm{O} 5-\mathrm{C} 10-\mathrm{H} 10 \mathrm{~A}$ & 117.5 \\
\hline $\mathrm{C} 6-\mathrm{C} 1-\mathrm{C} 2$ & $119.04(17)$ & $\mathrm{N} 1-\mathrm{C} 10-\mathrm{H} 10 \mathrm{~A}$ & 117.5 \\
\hline $\mathrm{C} 6-\mathrm{C} 1-\mathrm{C} 7$ & $120.53(16)$ & $\mathrm{N} 1-\mathrm{C} 11-\mathrm{H} 11 \mathrm{~A}$ & 109.5 \\
\hline $\mathrm{C} 2-\mathrm{C} 1-\mathrm{C} 7$ & $120.42(17)$ & $\mathrm{N} 1-\mathrm{C} 11-\mathrm{H} 11 \mathrm{~B}$ & 109.5 \\
\hline $\mathrm{C} 3-\mathrm{C} 2-\mathrm{C} 1$ & $120.22(17)$ & $\mathrm{H} 11 \mathrm{~A}-\mathrm{C} 11-\mathrm{H} 11 \mathrm{~B}$ & 109.5 \\
\hline $\mathrm{C} 3-\mathrm{C} 2-\mathrm{H} 2 \mathrm{~A}$ & 119.9 & $\mathrm{~N} 1-\mathrm{C} 11-\mathrm{H} 11 \mathrm{C}$ & 109.5 \\
\hline $\mathrm{C} 1-\mathrm{C} 2-\mathrm{H} 2 \mathrm{~A}$ & 119.9 & $\mathrm{H} 11 \mathrm{~A}-\mathrm{C} 11-\mathrm{H} 11 \mathrm{C}$ & 109.5 \\
\hline $\mathrm{C} 2-\mathrm{C} 3-\mathrm{C} 4$ & $119.58(15)$ & $\mathrm{H} 11 \mathrm{~B}-\mathrm{C} 11-\mathrm{H} 11 \mathrm{C}$ & 109.5 \\
\hline $\mathrm{C} 2-\mathrm{C} 3-\mathrm{C} 8$ & $121.87(16)$ & $\mathrm{N} 1-\mathrm{C} 12-\mathrm{H} 12 \mathrm{~A}$ & 109.5 \\
\hline $\mathrm{C} 4-\mathrm{C} 3-\mathrm{C} 8$ & $118.50(15)$ & $\mathrm{N} 1-\mathrm{C} 12-\mathrm{H} 12 \mathrm{~B}$ & 109.5 \\
\hline $\mathrm{C} 5-\mathrm{C} 4-\mathrm{C} 3$ & $121.09(17)$ & $\mathrm{H} 12 \mathrm{~A}-\mathrm{C} 12-\mathrm{H} 12 \mathrm{~B}$ & 109.5 \\
\hline $\mathrm{C} 5-\mathrm{C} 4-\mathrm{H} 4 \mathrm{~A}$ & 119.5 & $\mathrm{~N} 1-\mathrm{C} 12-\mathrm{H} 12 \mathrm{C}$ & 109.5 \\
\hline $\mathrm{C} 3-\mathrm{C} 4-\mathrm{H} 4 \mathrm{~A}$ & 119.5 & $\mathrm{H} 12 \mathrm{~A}-\mathrm{C} 12-\mathrm{H} 12 \mathrm{C}$ & 109.5 \\
\hline $\mathrm{C} 6-\mathrm{C} 5-\mathrm{C} 4$ & $118.27(17)$ & $\mathrm{H} 12 \mathrm{~B}-\mathrm{C} 12-\mathrm{H} 12 \mathrm{C}$ & 109.5 \\
\hline $\mathrm{C} 6-\mathrm{C} 5-\mathrm{C} 9$ & 121.07 (18) & & \\
\hline
\end{tabular}




\begin{tabular}{|c|c|c|c|}
\hline $\mathrm{O} 4^{\mathrm{i}}-\mathrm{Mn} 1-\mathrm{O} 1-\mathrm{C} 7$ & $-2.37(18)$ & $\mathrm{Mn} 1^{\mathrm{iv}}-\mathrm{O} 2-\mathrm{C} 7-\mathrm{O} 1$ & $-2.1(2)$ \\
\hline $\mathrm{O} 3{ }^{\mathrm{ii}}-\mathrm{Mn} 1-\mathrm{O} 1-\mathrm{C} 7$ & $-133.56(19)$ & $\mathrm{Mn} 1^{\mathrm{iv}}-\mathrm{O} 2-\mathrm{C} 7-\mathrm{C} 1$ & $-179.51(13)$ \\
\hline $\mathrm{O} 5-\mathrm{Mn} 1-\mathrm{O} 1-\mathrm{C} 7$ & $-29.3(4)$ & $\mathrm{Mn} 1-\mathrm{O} 1-\mathrm{C} 7-\mathrm{O} 2$ & $104.6(2)$ \\
\hline $\mathrm{O} 22^{\mathrm{iii}}-\mathrm{Mn} 1-\mathrm{O} 1-\mathrm{C} 7$ & $132.99(19)$ & $\mathrm{Mn} 1-\mathrm{O} 1-\mathrm{C} 7-\mathrm{C} 1$ & $-78.0(2)$ \\
\hline 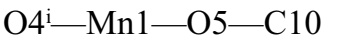 & $-152.61(17)$ & $\mathrm{C} 6-\mathrm{C} 1-\mathrm{C} 7-\mathrm{O} 2$ & $-178.90(18)$ \\
\hline $\mathrm{O} 3^{\mathrm{ii}}-\mathrm{Mn} 1-\mathrm{O} 5-\mathrm{C} 10$ & $-19.79(16)$ & $\mathrm{C} 2-\mathrm{C} 1-\mathrm{C} 7-\mathrm{O} 2$ & $2.0(3)$ \\
\hline $\mathrm{O} 1-\mathrm{Mn} 1-\mathrm{O} 5-\mathrm{C} 10$ & $-125.7(2)$ & $\mathrm{C} 6-\mathrm{C} 1-\mathrm{C} 7-\mathrm{O} 1$ & $3.7(3)$ \\
\hline 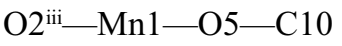 & $71.94(17)$ & $\mathrm{C} 2-\mathrm{C} 1-\mathrm{C} 7-\mathrm{O} 1$ & $-175.45(17)$ \\
\hline $\mathrm{C} 6-\mathrm{C} 1-\mathrm{C} 2-\mathrm{C} 3$ & $0.5(3)$ & $\mathrm{Mn} 1^{\mathrm{vi}}-\mathrm{O} 4-\mathrm{C} 8-\mathrm{O} 3$ & $-26.5(3)$ \\
\hline $\mathrm{C} 7-\mathrm{C} 1-\mathrm{C} 2-\mathrm{C} 3$ & $179.64(16)$ & $\mathrm{Mn} 1 \mathrm{vi}-\mathrm{O} 4-\mathrm{C} 8-\mathrm{C} 3$ & $155.59(13)$ \\
\hline $\mathrm{C} 1-\mathrm{C} 2-\mathrm{C} 3-\mathrm{C} 4$ & $0.8(3)$ & $\mathrm{Mn} 1^{\mathrm{v}}-\mathrm{O} 3-\mathrm{C} 8-\mathrm{O} 4$ & $-12.1(3)$ \\
\hline $\mathrm{C} 1-\mathrm{C} 2-\mathrm{C} 3-\mathrm{C} 8$ & $-176.42(16)$ & $\mathrm{Mn} 1 \mathrm{v}-\mathrm{O} 3-\mathrm{C} 8-\mathrm{C} 3$ & $165.74(13)$ \\
\hline $\mathrm{C} 2-\mathrm{C} 3-\mathrm{C} 4-\mathrm{C} 5$ & $-1.3(3)$ & $\mathrm{C} 2-\mathrm{C} 3-\mathrm{C} 8-\mathrm{O} 4$ & $-163.04(17)$ \\
\hline $\mathrm{C} 8-\mathrm{C} 3-\mathrm{C} 4-\mathrm{C} 5$ & $176.03(17)$ & $\mathrm{C} 4-\mathrm{C} 3-\mathrm{C} 8-\mathrm{O} 4$ & $19.7(2)$ \\
\hline $\mathrm{C} 3-\mathrm{C} 4-\mathrm{C} 5-\mathrm{C} 6$ & $0.4(3)$ & $\mathrm{C} 2-\mathrm{C} 3-\mathrm{C} 8-\mathrm{O} 3$ & $18.9(2)$ \\
\hline $\mathrm{C} 3-\mathrm{C} 4-\mathrm{C} 5-\mathrm{C} 9$ & $179.3(2)$ & $\mathrm{C} 4-\mathrm{C} 3-\mathrm{C} 8-\mathrm{O} 3$ & $-158.37(17)$ \\
\hline $\mathrm{C} 2-\mathrm{C} 1-\mathrm{C} 6-\mathrm{C} 5$ & $-1.4(3)$ & $\mathrm{Mn} 1-\mathrm{O} 5-\mathrm{C} 10-\mathrm{N} 1$ & $172.24(15)$ \\
\hline $\mathrm{C} 7-\mathrm{C} 1-\mathrm{C} 6-\mathrm{C} 5$ & $179.47(18)$ & $\mathrm{C} 11-\mathrm{N} 1-\mathrm{C} 10-\mathrm{O} 5$ & $179.4(2)$ \\
\hline $\mathrm{C} 4-\mathrm{C} 5-\mathrm{C} 6-\mathrm{C} 1$ & $0.9(3)$ & $\mathrm{C} 12-\mathrm{N} 1-\mathrm{C} 10-\mathrm{O} 5$ & $5.1(3)$ \\
\hline $\mathrm{C} 9-\mathrm{C} 5-\mathrm{C} 6-\mathrm{C} 1$ & $-178.0(2)$ & & \\
\hline
\end{tabular}

Symmetry codes: (i) $-x,-y+2, z+1 / 2$; (ii) $-x+1 / 2, y-1 / 2, z+1 / 2$; (iii) $x-1 / 2,-y+3 / 2, z$; (iv) $x+1 / 2,-y+3 / 2, z$; (v) $-x+1 / 2, y+1 / 2, z-1 / 2$; (vi) $-x,-y+2, z-1 / 2$. 